\title{
Outcome of meningococcal group B meningitis*
}

\author{
PETER D MOSS
}

SUMMARY Sixty children who survived meningitis during the outbreak of meningococcal group B $\vec{\circ}$ infection in Bolton 1971-74 were assessed between 5 and 9 years later. Each case, together with a matched control, has been examined clinically and subjected to a number of psychological tests and to routine audiology. The results, unlike those from other series, did not demonstrate any incoordination, ataxia, or other physical abnormality, nor was there any statistically significant impairment on psychological testing. The incidence of sensorineural deafness $(5 \%)$, although marginally lower, was comparable with the best of other series. When compared with the incidence in controls $(3 \%)$ it is not statistically significant. A 'mattress test', suggestive of vestibular damage, was positive in $\overrightarrow{0}$ those with more severe degrees of sensorineural deafness. The high mortality in the Bolton series 의 $(17 \%)$ has been reconsidered and it is concluded that unless a potent meningococcus type B vaccine is developed, mortality would still be high in a similar outbreak today.

Although there have been several follow-up studies of cases of meningitis since the war, these have concentrated on aspects such as audiology or psychology, rather than being comprehensive. Few studies have used controls.

In the present study 60 children who had meningococcal meningitis in the Bolton outbreak of 1970-74 were assessed after an interval of 5-9 years. Three types of investigation were done: clinical, audiological, and psychological. A similar number of controls was subjected to exactly the same investigations. This was considered essential as possible complications of meningitis-such as deafness, behavioural problems, and various degrees of brain damage-occur for other reasons.

The Bolton outbreak was reported by Farries et al. ${ }^{1}$ There were 82 cases of meningococcal infection including $14(17 \%)$ children who died. Seventy-three of these children were admitted to one hospital. Thirty-three were under age 2 years and all but 6 of the others were under 7 years. Meningococci were isolated from the cerebrospinal fluid, the blood, or both these in 54 cases. No further diagnostic criteria were given in the original paper but all the case records have been examined (by P D M) to ascertain that the 60 cases in the present series are correctly included, having regard to the history, the clinical findings including the typical rash in many cases, the laboratory findings, and the response to treatment.

Thirty-nine strains of meningococci were tested

*Carried out on behalf of The Medical Services Study Group of the Royal College of Physicians, Albert Street, London. and 32 were found to be group B. There were also 3 group A, 3 group $C$, and one that was untypable. All but two strains, one each group $A$ and $C$, were sulphonamide sensitive. All the group B strains were found to be of serotype 2 .

The attack rate in children was high and reached 184 per 100000 in the 6- to 11 -month age group. Thirty-four per cent of family contacts carried the organism in the nasopharnyx. Several additional cases not included in the original report have been found and included in this assessment.

\section{Material}

At the time of the illness the children were aged between 1 month and 7 years 10 months. There were 34 boys and 26 girls. Controls were chosen with the $\delta$ help of the head teachers. As far as possible a child of the same gender, from the same class, and nearest $ᄋ$ in age to the child with meningitis was chosen. In the few instances that this was not possible, the next nearest was accepted. Both groups came, with $\bar{N}$ very few exceptions, from social classes IV and V. Although this method of selection of controls is $\tilde{O}$ open to criticism, after considerable deliberation $N$ it was considered the best for this particular in- $O$ vestigation. Similar controls were used by Redlin and von Ungern-Sternberg ${ }^{2}$ in part of their study.

\section{Method}

Each child was examined clinically (by P D M). The accompanying relative (generally the mother) was $\frac{\vec{Q}}{\mathscr{Q}}$ 
asked for details of the child's health before and after the illness. He was then weighed and measured and the result plotted on Tanner and Whitehead 0-19 charts. His near and distant vision was assessed using Snellen types and a sample of his urine was tested. A full clinical examination was then carried out, including a standard neurological assessment. The fundi were examined but mydriatics were not used. Special care was taken to exclude ataxia or incoordination.

All case notes were examined and details of illness, treatment, and progress obtained.

Audiology. Audiological examination was carried out on a second visit by a member of the team employed in school assessments. Four audiologists used standard equipment, commonly a Kamplex machine. Tympanograms were done if it was suspected that middle-ear disease was interfering with free movement of the tympanic membrane. Masked bone conduction tests were done in those found to be deaf. Those at first thought to be defective in hearing were reassessed at a later date, as is the usual practice, and some were then regarded as having normal hearing. Those children who exhibited sensorineural deafness were subjected to a 'mattress test', as described by Landthaler and AndrieuGuitrancourt, ${ }^{3}$ in order to seek vestibular damage. The subject is asked to stand steadily, walk forwards, turn, and then walk backwards on a length of thick latex foam, first with the eyes open and then blindfolded. Being deprived of proprioceptive information from the feet and of vision he is dependent on his vestibular apparatus for balance. A count is made of losses of balance and lurches.

Psychological assessments. These were carried out by one of four education psychologists. The Wechsler intelligence scale for children (revised) (WISC-R) was used (omitting the subtest for comprehension), and the Burt reading test. These tests cover a wide variety of tasks each of which is standardised for age. The IQ scores may be directly compared for children of different ages and can be related to the commonly used Standford-Binet test.

Statistical methods. The $\chi^{2}$ test and Fisher's exact test were used. ${ }^{4}$

\section{Results}

There was no significant difference in the findings on clinical examinations between the meningitis and the control groups. The distribution curves for both height and weight were very similar. In each group there were 4 children with squints. Eight meningitis cases had vision worse than $6 / 9$ or N5 compared with 10 controls. Thirteen meningitis cases performed poorly when taste was tested compared with 19 of the controls. One meningitis case demonstrated a poor sense of smell compared with 7 controls. There was one meningitis case with nystagmus and none in the controls. One control had a tremor of the hands and exaggerated knee jerks. There was no such finding in the meningitis group, and no case of ataxia or incoordination in either group. Ten of the meningitis cases had perfect or nearly perfect teeth compared with 12 controls. No abnormality relevant to the present work was found in other systems.

Audiology. The results are set out in Table 1. During the history taking each parent was questioned about any ear troubles from which her child might have suffered. Fourteen of the cases and 17 of the controls gave a positive reply. On initial assessment 13 of the meningitis group were found to have some hearing impairment compared with 7 of the controls. On repeat testing this was reduced to $6(10 \%)$ and 4 $(7 \%)$ respectively. Bone conduction tests were unsuccessful in one control owing to poor co-operation. Three $(5 \%)$ of the meningitic group showed sensorineural deafness, all unilateral, as did $2(3 \%)$ controls, one of which was unilateral. These children were then asked to perform the mattress test. One child in each group showed no unsteadiness. Each had only minimal nerve loss. The remaining 2 cases and one control were unsteady and all had appreciable sensorineural deafness.

Psychological testing. Table 2 sets out the average scores obtained in psychological tests. In no case is the difference of the mean scores significant in statistical terms ( $\chi^{2}$ test). In the verbal tests and the full-scale IQ test however, there are significant

\section{Table 1 Cases and controls with hearing loss}

\begin{tabular}{|c|c|}
\hline Cases & Hearing loss \\
\hline 1 & Unilateral conductive loss maximum $40 \mathrm{~dB}$ in low tones \\
\hline 2 & Minimal unilateral sensorineural loss \\
\hline 3 & $\begin{array}{l}\text { Moderate unilateral sensorineural loss. Maximum air } \\
50 \mathrm{~dB} \text { and bone } 35 \mathrm{~dB}\end{array}$ \\
\hline 4 & Minimal unilateral conductive loss \\
\hline 5 & $\begin{array}{l}\text { Unilateral sensorineural loss. Maximum air } 50 \mathrm{~dB} \text { and } \\
\text { bone } 60 \mathrm{~dB}\end{array}$ \\
\hline 6 & Unilateral conductive loss. Maximum $55 \mathrm{~dB}$ \\
\hline \multicolumn{2}{|l|}{ Controls } \\
\hline 1 & Unilateral conductive loss. Maximum $45 \mathrm{~dB}$ in low tones \\
\hline 2 & Unilateral conductive loss. Maximum $50 \mathrm{~dB}$ in high tones \\
\hline 3 & $\begin{array}{l}\text { Bilateral loss, probably sensorineural. Bone conductive } \\
\text { tests impracticable. Maximum loss right } 50 \mathrm{~dB} \text {, left } \\
45 \mathrm{~dB} \text {. }\end{array}$ \\
\hline 4 & Unilateral minimal sensorineural loss \\
\hline
\end{tabular}


Table 2 Results of psychological assessments

\begin{tabular}{|c|c|c|c|}
\hline & \multicolumn{2}{|c|}{ Average scores } & \multirow[b]{2}{*}{ Statistical result } \\
\hline & Cases & Controls & \\
\hline \multicolumn{4}{|l|}{$\begin{array}{l}\text { Verbal tests } \\
\quad(W I S C-R)\end{array}$} \\
\hline Information & $8 \cdot 4$ & $8 \cdot 1$ & NS Variances differ \\
\hline Similarities & $8 \cdot 6$ & $8 \cdot 9$ & NS Variances differ \\
\hline Arithmetic & 9.9 & $9 \cdot 8$ & NS Variances differ \\
\hline Vocabulary & $8 \cdot 9$ & 9.0 & NS Variances differ \\
\hline Digital span & $8 \cdot 7$ & $8 \cdot 7$ & NS \\
\hline Verbal test IQ & $93 \cdot 0$ & $93 \cdot 5$ & NS Variances differ \\
\hline \multicolumn{4}{|l|}{$\begin{array}{l}\text { Performance tests } \\
\quad(W I S C-R)\end{array}$} \\
\hline Picture completion & $10 \cdot 0$ & $9 \cdot 2$ & NS \\
\hline Block design & 9.8 & $10 \cdot 2$ & NS \\
\hline Object assembly & $9 \cdot 8$ & $10 \cdot 4$ & NS \\
\hline Coding & $8 \cdot 5$ & $8 \cdot 6$ & NS \\
\hline Performance scale IQ & $96 \cdot 0$ & $98 \cdot 2$ & NS \\
\hline Full-scale IQ & $94 \cdot 0$ & $95 \cdot 1$ & NS Variances differ \\
\hline \multicolumn{4}{|l|}{ Burt reading test } \\
\hline \multicolumn{4}{|l|}{ Chronological age } \\
\hline & $10 \cdot 9$ & $10 \cdot 7$ & NS \\
\hline \multicolumn{4}{|l|}{ Reading quotient } \\
\hline (mean) & $93 \cdot 0$ & $93 \cdot 2$ & NS \\
\hline
\end{tabular}

differences between the variances in distribution between cases and controls.

Cross analyses. The following additional analyses were done comparing each with (1) hearing defect, and (2) psychological tests (Table 2).

(a) The severity of the illness as judged by the presence of marked drowsiness, unconsciousness, or convulsions.

(b) The duration of the fever. (c) The recurrence of fever.

(d) Underweight.

(e) Overweight.

(f) Short stature.

(g) Tall stature.

(h) The possible ill effects of each of the following drugs: sulphadiazine, streptomycin, kanamycin, and chloramphenicol.

(i) The duration of the illness of more than one day before admission to hospital, and (j) before being given any treatment.

In each calculation Fisher's exact test was used for hearing and the $\chi^{2}$ for psychological tests. No positive correlations were found.

\section{Discussion}

The outcome in meningitis depends on a number of variable factors. Among these are the causative organisms, the age of the patient, the medical era when it occurs, the type of community, the general level of nutrition and child health, and the efficiency of the medical services.

Bolton is a northern industrial town. In the early 1970 s, at the time that these meningitis cases were occurring, most of the housing dated from the industrial revolution. There is no reason to believe that medical care was either particularly good or especially bad. The infant mortality rate in 1970 in the old county borough of Bolton (population 152 000) was $22 \cdot 12$. In 1980 in the new Bolton Health Authority area (population 260200 ) the infant mortality rate was $12 \cdot 00$. The corresponding figures for England and Wales were 18.00 and 12.00 respectively. The immigrant population was low and the series contained only one child from the Indian subcontinent. Social Classes IV and V were the ones to be affected. The carrier rate for meningitis group B was high ( $34 \%$ ) among families of cases. The death rate was high $(17 \%)$, and the children who succumbed were all aged under 2 years. It is reasonable to suppose that had they been in better general health they might have been better able to withstand the infection.

It is noteworthy that the amount of morbidity in survivors is very little. There was nothing at all to pick out on general examination and there is only a small excess of hearing loss in the meningitis cases compared with controls.

Similarly psychological testing showed no sequelae that could clearly be attributed to meningitis. The worst that could be said was that there might be a 'tendency towards' lower performance and lower IQ without it being in any way statistically significant compared with controls.

There are few postwar papers that deal generally with the long-term ill effects of meningitis. Grove and Fox ${ }^{5}$ dealt with meningococcal meningitis and found that a number of minor sequelae-such as headaches, muscle, and joint pains-frequently persisted for more than a year. Sproles et al. ${ }^{6}$ found that $10 \%$ of 40 cases of Haemophilus influenzae meningitis were severely brain damaged, that $27.5 \%$ had clear residual handicap, and only $45 \%$ completely recovered. Alon et al. ${ }^{7}$ found that 15 of 72 cases of 'septic meningitis' had major neurological sequelae and that 19 of 72 cases had minimal brain damage.

Fitzhardinge et al. ${ }^{8}$ studied 18 survivors of neonatal meningitis and found that only 2 had physical disabilities: one exaggerated reflexes and one hypotonia. Landthaler and Andrieu-Guitrancourt ${ }^{3}$ studied 53 cases of meningitis of various types and found that 9 cases were ataxic and had hearing defects. In none of these studies was a control group used.

In the present series there was no significant higher incidence of any physical disability compared with controls. In particular there was no ataxia as described by some authors. 
The incidence of hearing loss after meningitis varies greatly in different reports. There are four series dealing with meningococcal cases. Grove and Fox found deafness in $12.3 \%$ of cases. Teng YuanCh'Eng et al. ${ }^{9}$ reviewed 337 cases of deafness after meningococcal meningitis and found it to be bilateral in $95 \%$. Habib et al.$^{10}$ reviewed 775 cases and found $45(5.8 \%)$ to have impaired hearing. Of these it was complete in 18 and partial in 26. Trolle, 11 finding a similar incidence, found that most cases were sensorineural in type. Controls were not used in any of these series.

In two studies of $H$. influenzae meningitis the incidence is higher. Svenungsson et al ${ }^{12}$ found hearing loss in $23 \%$ and Rosenhall et al. ${ }^{13}$ found hearing loss in $18.1 \%$.

Other series deal with groups of meningitis caused by various bacteria. Keane et al. ${ }^{14}$ found $6 \%$ to be deaf. $\mathrm{Nadol}^{15}$ reported an incidence of $21 \%$ in 110 cases, yet Fitzhardinge et al. ${ }^{8}$ did not find any hearing loss in 37 cases of neonatal meningitis. Redlin and von Ungern-Sternberg ${ }^{2}$ used controls with 25 cases of purulent meningitis and found 7 cases of hearing loss in the cases and 3 in the controls.

In the present series the incidence of hearing loss is $10 \%$ in the meningitis cases and $7 \%$ in the controls. Half of these were sensorineural in type in both groups, the incidence therefore being $5 \%$ and $3 \%$ respectively. In one case and one control the nerve loss was slight, and in 2 cases and one control it was significant. The figures are somewhat lower than in the various series quoted, but as they are controlled they probably reflect more correctly the true incidence. It suggests that deafness is not common as a sequel to meningococcal meningitis which is adequately treated and that the incidence is lower than in other forms of bacterial meningitis. Streptomycin had not been used in the treatment in any child with hearing loss in this series.

The mattress test by itself cannot be accepted as indicating vestibular damage. This would require additional specific tests. It was positive in this series only in those with more severe degrees of sensorineural deafness, although in 2 out of the 3 it was unilateral. In the control child it was bilateral but there were indications of other neurological damage which probably occurred at birth. It suggests that cases of sensorineural deafness after meningitis should in future be investigated for vestibular damage also.

As with other sequelae of meningitis there is a wide divergence in the findings of different workers when considering psychological problems. In contrast with other workers, psychologists usually used controls. Unfortunately, none followed up cases of meningococcal infection alone. There are one or two studies of $H$. influenzae meningitis but all others are of groups of cases of meningitis of various aetiology. Sproles and Azerrad ${ }^{6}$ studied 40 children who had suffered from $\boldsymbol{H}$. influenzae meningitis and found that problems persisted in $27.5 \%$. These were mild depression of $\mathrm{IQ}$, poor school performance, slight brain damage, or behavioural problems. Sell et al. ${ }^{16}$ used controls when studying 'bacterial' meningitis cases. In one group of 21 children they used siblings as controls and WISC tests were performed. The mean IQ in the cases was 86 and in the controls it was 97 . In a second series they used children 'considered to be normal' as controls and a different series of tests. They concluded that all the indications were that the meningitic group functioned at significantly lower levels than the controls although the figures were not statistically significant. Redlin and von Ungern-Sternberg ${ }^{2}$ also studied 25 cases of purulent meningitis and 25 controls. They found that the IQ was within the normal range but showed some disabilities in perception, oculo-motor control, alertness, and reflex response. These results were not statistically significant. Alon et al. ${ }^{7}$ studied septic meningitis cases and found that 19 out of 72 suffered from minimal brain damage. Controls were not used. Fitzhardinge et al. ${ }^{8}$ studied 18 cases after neonatal meningitis. The mean IQ was 90 which is within the normal range, but they considered that there was a tendency towards lower average intelligence after neonatal meningitis. There was no difference between the results of verbal and performance tests in the WISC series that might indicate 'minimal brain damage' but there was a tendency to disturbances of expression and to speech problems. Controls were not used.

In the present series there was no difference which was statistically significant between the cases and controls in the scores obtained on WISC-R tests and Burt reading tests. It is noted however, that in the performance scale there is a $2 \cdot 2$ points difference in the mean scores in favour of controls. In the fullscale IQ the difference is 1.2 points in favour of controls. This might be construed as a 'tendency towards' lower performance and lower IQ. Although there were no significant differences in means, scores in the individual subtests-information, similarities, vocabulary verbal IQ, and full-scale IQ-there were significant differences in the frequency polygons. These differences tended to indicate that the controls scored more often in the middle range. A difference between scoring in the performance group and the verbal group of tests is often taken as one pointer towards minimal brain damage. The mean scores in these two groups in our series were 
almost exactly the same: 11.2 and 11.5 respectively. Other criteria commonly considered are perceptual difficulties and poor motor-visual coordination, scatter on WISC subtests, and differences in IQ. There was no clear evidence for these in the present series from the tests employed. Some other workers have resorted to more complicated tests, although generally they have not produced results which are statistically significant.

Taken as a whole the investigation shows a remarkable lack of serious sequelae after meningococcal meningitis. There is no physical abnormality of note and only a slightly lower level of hearing loss than in the controls. There is probably some vestibular damage associated with sensorineural deafness if it is more than minimal. Psychological problems at the very worst can only be put as a slight tendency towards lower scores on the performance scale and in full IQ. They are very far from being statistically significant.

It is apparent that in this outbreak treatment was prompt and efficient, aided by early diagnosis by general practitioners. This was probably assisted by a high level of awareness as the number of cases increased in the outbreak. It may be thought that the low incidence of residual disease was in part due to a large number of deaths having eliminated the most serious cases. This was not so because only one of the 14 deaths in the outbreak of meningococcal infections was due to meningitis and the child was dead on arrival at hospital. The others were attributed to septicaemia with adrenal haemorrhage. Virtually all the cases of meningitis who survived and those now at school have very little residual handicap.

It is perhaps fortunate that it was the meningococcus which was the organism endemic in Bolton rather than one of the other bacteria that can cause meningitis. As can be inferred from the review of the literature the morbidity, although perhaps not the mortality, tends to be appreciably worse in the other types of meningitis infection. The outcome with modern treatment is usually better than it was 30 to 40 years ago, but that is not always so. It depends on the part of the world in which it occurs and the quality of the medical services. As recently as 1979, Alon et al. ${ }^{7}$ reported 15 of 72 cases of 'septic meningitis' with major neurological sequelae. Yet in 1974 in Toronto Fitzhardinge et al.$^{8}$ found practically no serious sequelae in 18 cases of neonatal meningitis of various types. Even between 30 and 40 years ago the results could be good, as in the series reported by Trolle ${ }^{11}$ from Sweden in 1950.

The cross tabulation studies gave no positive information.

The mortality in the Bolton 1971-74 outbreak of meningococcal type $\mathrm{B}$ infection was high $(17 \%)$. Could we do better today? Only one death was due to meningitis. All the others were due to septicaemia with adrenal haemorrhage and death was quick. The age range was 6 weeks to 2 years. None was from a household with a recognised meningococcal infection and all were from social classes IV or V. They occurred during a period of nearly $3 \frac{1}{2}$ years. Bolton was not isolated in an epidemiological sense as neighbouring towns in this densely populated part of the north west were also experiencing a higher than normal incidence of meningococcal type B infection. Doubtless some limited benefit would already be apparent as a result of better general health, better housing, and a reduction in overcrowding, but something producing more immediate results is required. Chemoprophylaxis in the circumstances cited would be both difficult to apply and expensive although it could reduce the incidence of infection. Vaccination in type $\mathrm{A}$ and $\mathrm{C}$ outbreaks has been used successfully in Nigeria on children aged between 5 and 15 years. ${ }^{17}$ All the Bolton children who died were much younger. There is unfortunately, no type $B$ vaccine yet available and until such time as it is successfully developed it is difficult to foresee much lowering of mortality in an outbreak of the Bolton type. Even when it becomes available it will have to be used selectively, with booster dosage in the very young and with chemoprophylaxis to cover the period up to the time immunity has developed. ${ }^{18}$

We thank Sir Cyril Clarke, Dr George Whitfield, and Dr J S Farries for constructive criticism. The Bolton Education Authority and the following staff members of the Bolton Area Health Authority helped with work in the field: Dr J S Farries, community physician; Mrs D Allen, Mrs O Hart, Mrs J Edmundson, and Mrs W Reece, audiologists; most of the health visitors and school nurses; Messrs G Williams, J Ashbrook, Y Aplin, and D Fergusson, education psychologists; the head teachers of most Bolton schools; Mrs J I Danson, typist; Mrs J Howarth, secretary. The statistical analyses were done in the Department of Statistics of the North Western Regional Health Authority by Mr K W Johnson and Miss L M Halsall. David Jakubovic translated French and German papers.

\section{References}

1 Farries J S, Dickson W, Greenwood E, Malhotra T R, Abbott J D, Jones D M. Meningococcal infections in Bolton 1971-74. Lancet 1975; ii: 118-20.

2 Redlin W, von Ungern-Sternberg U. Leistungsdefekte als Dauershäden nach eitriger Meningitis im Säuglingsalter. Prax Kinderpsychol Kinderpsychiatr 1972; 21 : 49-54. 
${ }^{3}$ Landthaler G, Andrieu-Guitrancourt J. Ataxies et surdités secondaires aux méningites purulentes de l'enfant; role de l'atteinte labyrinthique. Arch Fr Pediatr 1975; 32: 319-33.

4 Kendall M G, Stuart A. Advanced theory of statistics, third edition. Vol. 2. High Wycombe: Griffin, 1973: 570-2.

5 Grove W E, Fox M J. Postmeningitic complications with particular reference to otologic sequelae. Ann Otol Rhinol Laryngol 1949; 58: 771-88.

6 Sproles E T, Azerrad J, Williamson C, Merrill R E. Meningitis due to Hemophilus influenzae: long term sequelae. J Pediatr 1969; 75: 782-8.

7 Alon U, Naveh Y, Gardos M, Friedman A. Neurological sequelae of septic meningitis. Isr J Med Sci 1979; 15: 512-7.

8 Fitzhardinge $\mathbf{P}$ M, Kazemi M, Ramsay $\mathbf{M}$, Stern L. Long term sequelae of neonatal meningitis. Dev Med Child Neurol 1974; 16: 3-10.

9 Teng Yuan-Ch'Eng, Liu Juei-Hua, Hsu Yin-Hsiang. Meningitis and deafness. Chin Med J (Engl) 1962; 81: 127-30.

10 Habib R G, Girgis N I, Yassin M W, Laughlin L W, Sippel J E, Edman D C. Hearing impairment in meningococcal meningitis. Scand J Infect Dis 1979; 11: 121-3.

11 Trolle E. Defective hearing after meningococcal meningitis. Acta Oto Laryngol (Stockh) 1950; 38: 384-402.
12 Svenungsson B, Bengtsson E, Fluur E, Siegborn J. Hearing loss as a sequel to chloramphenicol and ampicillin treatment of Haemophilus influenzae meningitis. Scand J Infect Dis 1976; 8: 175-80.

13 Rosenhall U, Nylén O, Lindberg J, Kankkunen A. Auditory function after Haemophilus influenzae meningitis: two controlled studies. Acta Otolaryngol (Stockh) 1978; 85: 243-7.

14 Keane W M, Potsic W P, Rowe L D, Konke D F. Meningitis and hearing loss in children. Arch Otolaryngol 1979 ; 105: 39-44.

15 Nadol J B, Jr. Hearing loss as a sequelae of meningitis. Laryngoscope 1978; 88: 739-55.

${ }_{16}$ Sell S H W, Webb W W, Pate J E, Doyne E O. Psychological sequelae to bacterial meningitis. Pediatrics 1972; 49: 212-7.

17 Mohammed I, Zaruba K. Control of epidemic meningococcal meningitis by mass vaccination. Lancet 1981; ii: $80-3$.

18 Anonymous. Who should be given meningococcal vaccine? Lancet 1978; ii: 1185-6.

Correspondence to Dr P D Moss, 9/10 Briers Brow, Wheelton, Chorley, Lancs PR6 8HD.

Received 31 March 1982

\section{The following articles will appear in future issues of this journal:}

Paediatric manpower

$M$ Liberman and $M H$ Bellman

Enteropathy complicating maintenance therapy in acute lymphoblastic leukaemia

IJ Lewis, D Mainwaring, and J Martin

Variation in the composition of breast milk during the first 5 weeks of lactation: implications for the feeding of preterm infants

C M Hibberd, O G Brooke, N D Carter, M Haug, and G Harzer

Two-year study of the causes of postperinatal deaths classified in terms of preventability E M Taylor and $J$ L Emery

Phenobarbitone dosage in neonatal convulsions

$R$ A Ouvrier and R Goldsmith

Natural history of egg hypersensitivity

$R P K$ Ford and $B$ Taylor

Paediatric continuous ambulatory peritoneal dialysis

E J Eastham, H Kirpalani, D Francis, $R$ Gokal, and $R$ H Jackson 